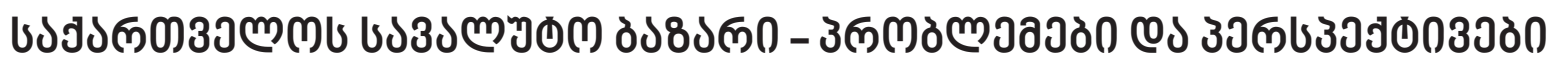

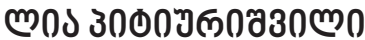

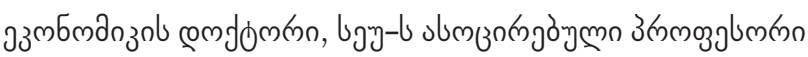

https://doi.org/10.35945/gb.2017.04.012

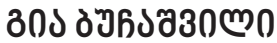

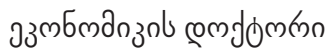

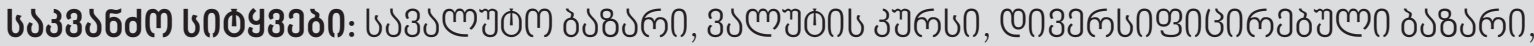

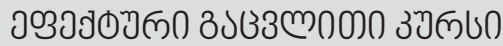

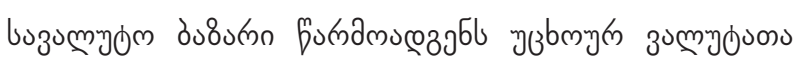

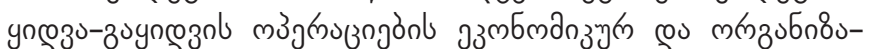

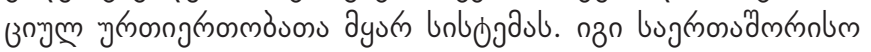

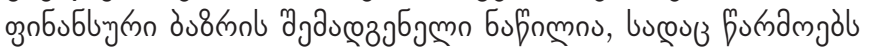

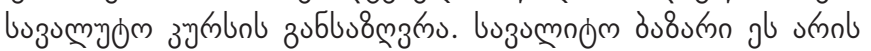
moुnढ̧

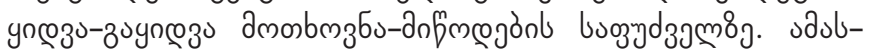

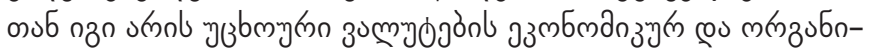

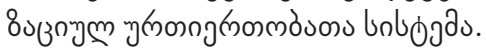

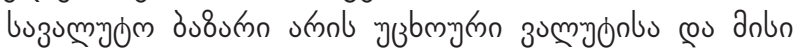

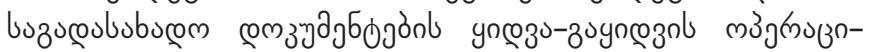

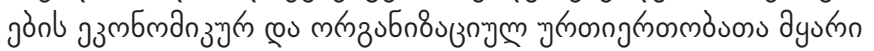
unbogas.

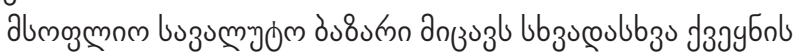
as

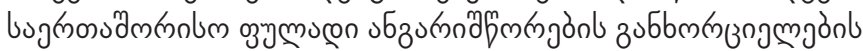
ajงubnoab.

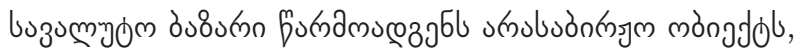

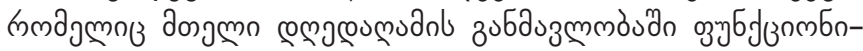

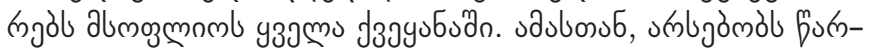

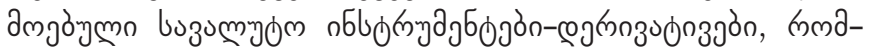

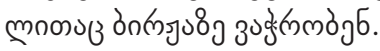

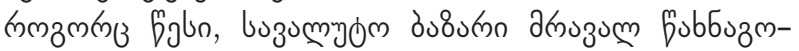

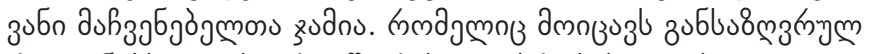

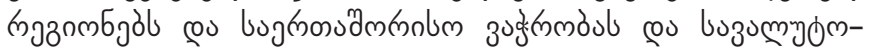

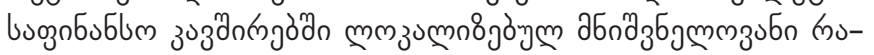

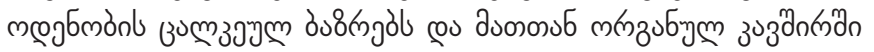

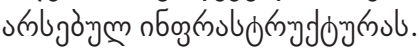

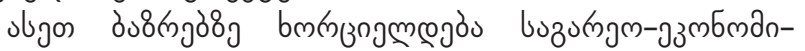

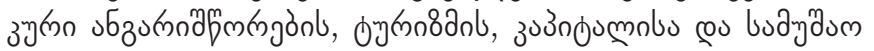

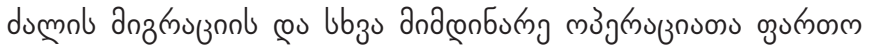

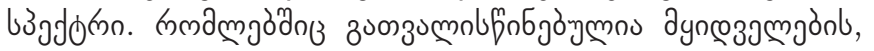

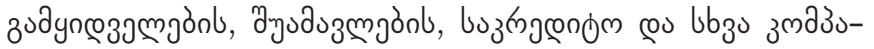

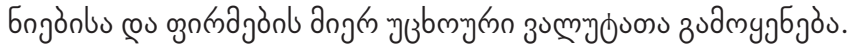

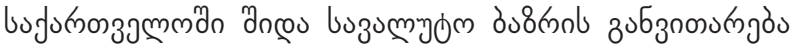

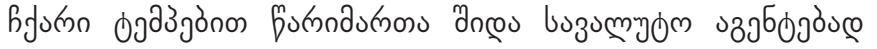
bufurnos зjмm

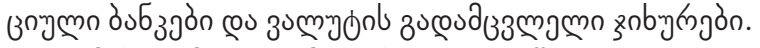

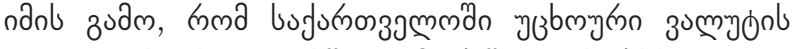

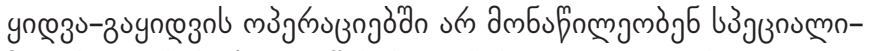

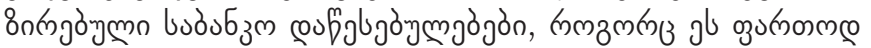

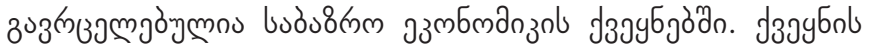

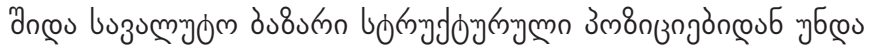

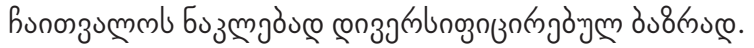

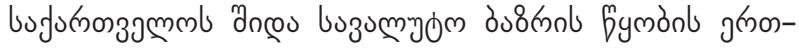

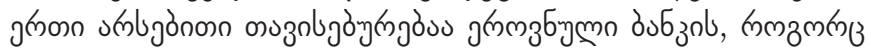

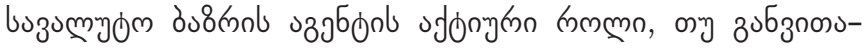

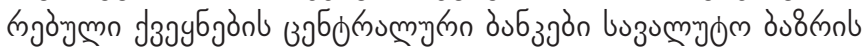

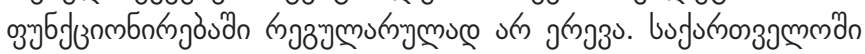

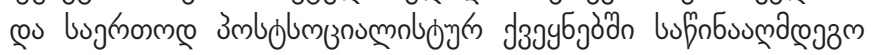

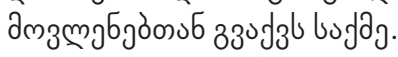

3u

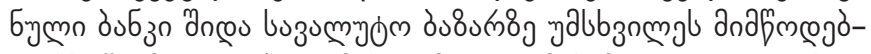
madl fujamuœ

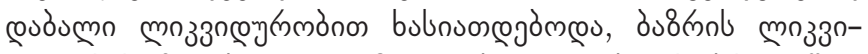

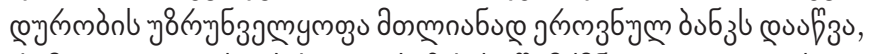

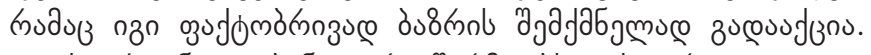

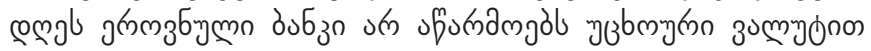

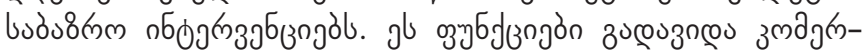

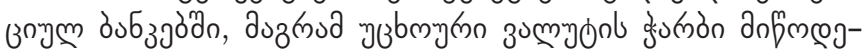
onb aganb3azudn aynœ

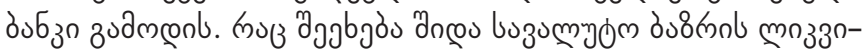

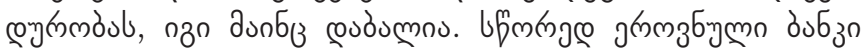
nбu nh hy

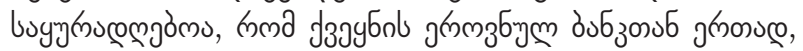

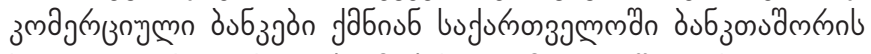

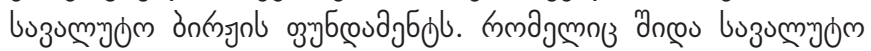
उu8

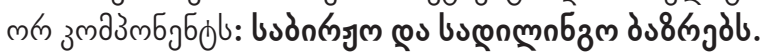

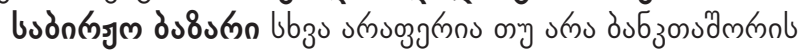

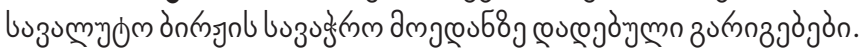

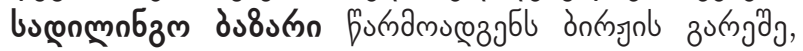

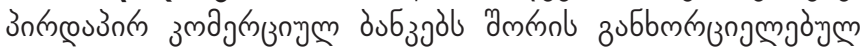

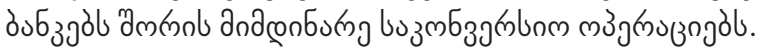

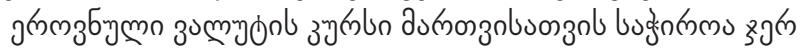
च

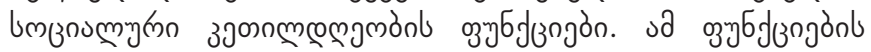

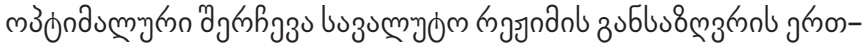

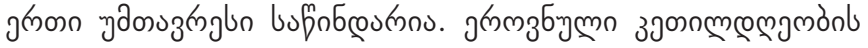

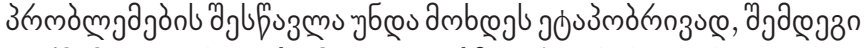

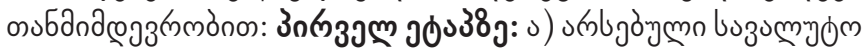

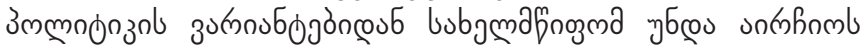

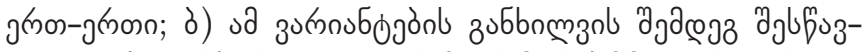

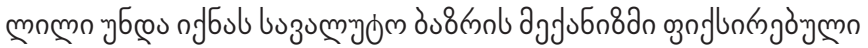




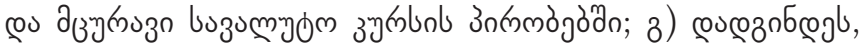

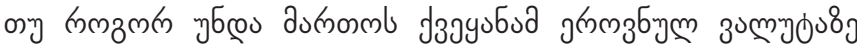

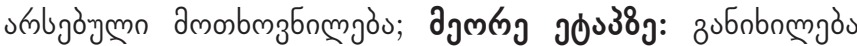

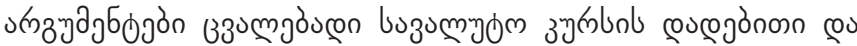

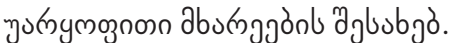

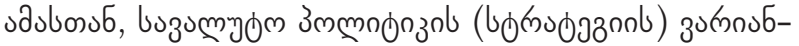

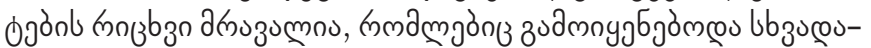

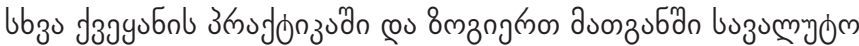

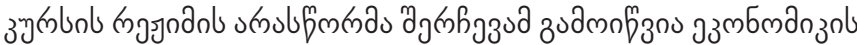

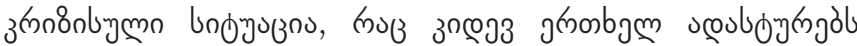

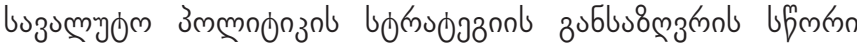

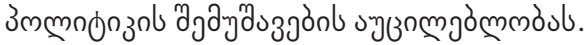

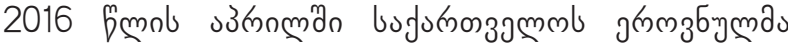

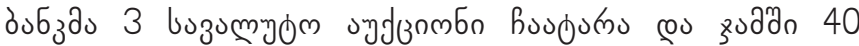

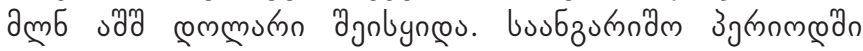

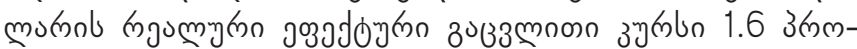

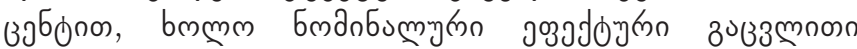

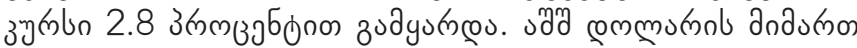

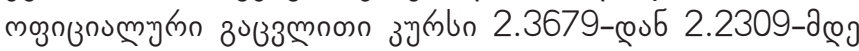

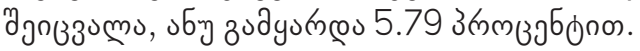

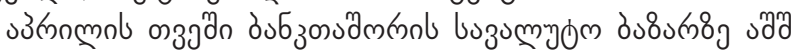

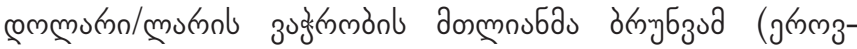

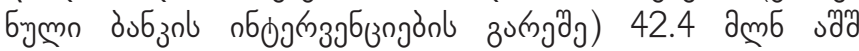

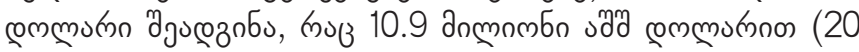

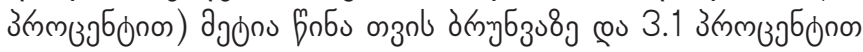

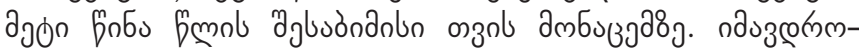

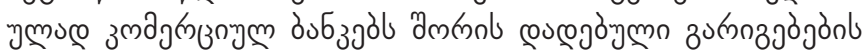

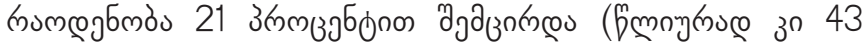

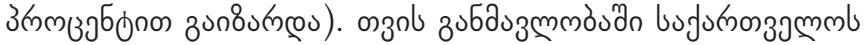

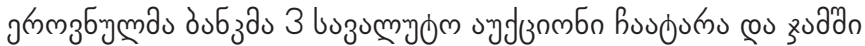

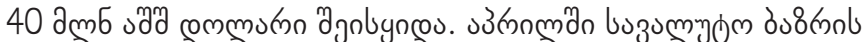

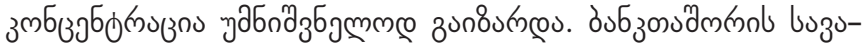

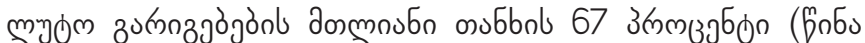

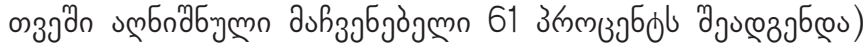

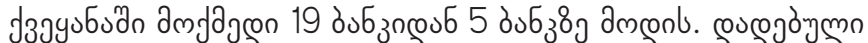

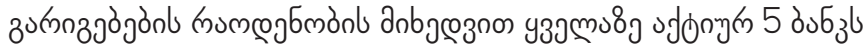

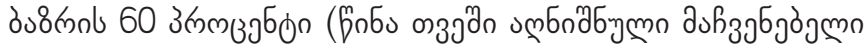

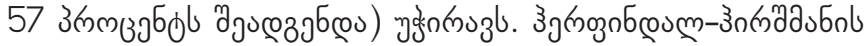

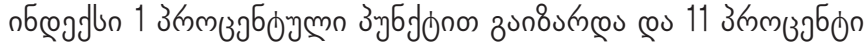

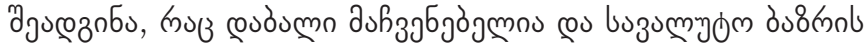

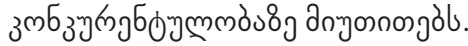

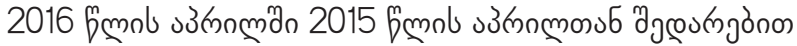

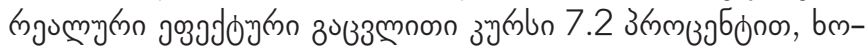

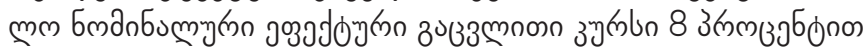

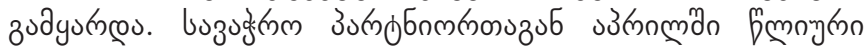

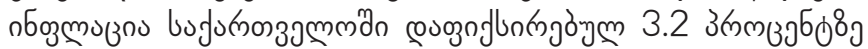

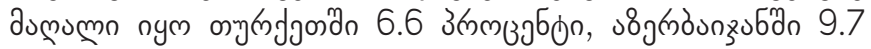

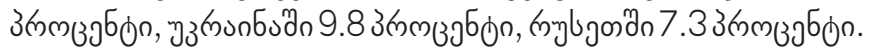

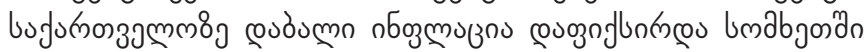

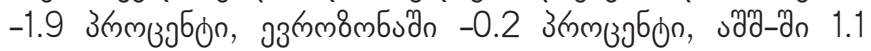

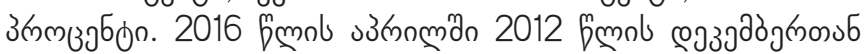

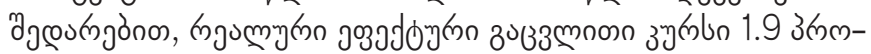

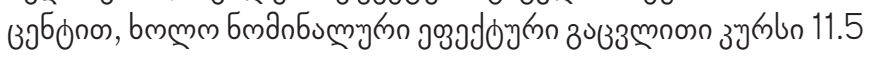

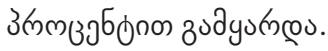

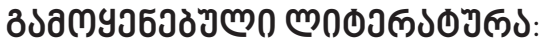

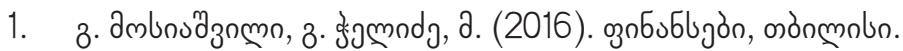

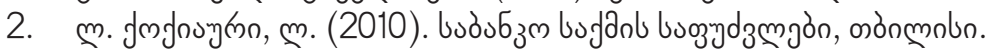

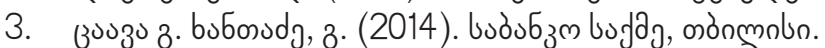

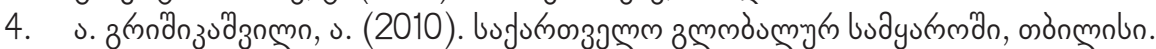




\section{GEORGIAN FOREIGN EXCHANGE MARKET - PROBLEMS AND PROSPECTS}

\section{LIA PITIURISHVILI}

https://doi.org/10.35945/gb.2017.04.012

Doctor of Economics, Associated Professor of Georgian National University, Georgia

\section{GIA BUCHASHVILI}

Doctor of Economics, Associated Professor of Georgian National University, Georgia

KEYWORDS: CURRENCY MARKET, CURRENCY EXCHANGE, DIVERSIFIED MARKET, EFFECTIVE EXCHANGE RATE

\section{SUMMARY}

This article deals with the foreign exchange market, exchange market can be considered as a less diversified marits essence and meaning in the world and in Georgia. Wide ket. Necessity of elaboration of the correct foreign exchange spectrum of foreign-economic settlements, tourism, capital, policy should be defined. For the purposes of mitigation of and labor migration, as well as other ongoing operations the strong fluctuation of the domestic currency, the currency are carried out on foreign currency markets. It envisages us- market and the National Bank of Georgia should jointly workage of foreign currencies by buyers, sellers, intermediaries, out the strategy which would allow the national currency to and other financial companies and firms. Georgian foreign maintain its stability. 